Mens

Revue d'histoire intellectuelle et culturelle

mens

\title{
Quarante ans de pacification tranquille : du projet de reconnaissance politique de la commission Laurendeau-Dunton aux politiques de protection des francophones en situation minoritaire
}

\section{Christophe Traisnel et Anne-Andrée Denault}

Volume 14-15, numéro 2-1, printemps-automne 2014

La Commission royale d'enquête sur le bilinguisme et le biculturalisme a 50 ans : période révolue ou projet inachevé ?

URI : https://id.erudit.org/iderudit/1035528ar

DOI : https://doi.org/10.7202/1035528ar

Aller au sommaire du numéro

\section{Éditeur(s)}

Centre de recherche en civilisation canadienne-française

ISSN

1492-8647 (imprimé)

1927-9299 (numérique)

Découvrir la revue

Citer cet article

Traisnel, C. \& Denault, A.-A. (2014). Quarante ans de pacification tranquille : du projet de reconnaissance politique de la commission Laurendeau-Dunton aux politiques de protection des francophones en situation minoritaire. Mens, 14-15(2-1), 89-129. https://doi.org/10.7202/1035528ar

\section{Résumé de l'article}

Nous souhaitons montrer dans ce texte que l'État canadien semble, en fait, s'être prévalu d'une sorte de "droit d'inventaire " quant aux débats, aux réflexions et aux propositions formulés dans le cadre de la Commission royale d'enquête sur le bilinguisme et le biculturalisme, en choisissant de s'attarder davantage à trouver des solutions pour régler la « crise " politique que traversait alors le Canada, plutôt qu'en en suivant les recommandations. Ce faisant, si les conflits linguistiques au Canada se sont peu à peu pacifiés, un problème reste en suspens : celui de la reconnaissance des francophonies canadiennes. Il ne s'agit pas de proposer ici une analyse historique de la portée et des limites des travaux de la Commission, mais plutôt de comprendre l'influence de ses travaux sur les fondements du « modèle canadien de reconnaissance " à partir desquels sont pensées les principales politiques destinées aux communautés francophones en situation minoritaire (CFSM). Pour ce faire, nous chercherons à cerner la place relativement modeste qu'occupe la question de la reconnaissance politique dans une littérature pourtant dense consacrée aux communautés francophones en situation minoritaire, puis nous resituerons la Commission dans les débats identitaires qui traversaient à l'époque le Canada français. Nous montrerons ensuite que le législateur canadien s'est plutôt engagé dans une politique de protection des francophones en situation minoritaire, plutôt que dans une politique de reconnaissance de ces communautés. Cela nous permettra, enfin, en menant une réflexion plus prospective sur le " modèle " canadien, de nous attarder sur les conséquences de ces politiques sur les francophonies canadiennes elles-mêmes. 


\title{
Quarante ans de pacification tranquille : du projet de reconnais- sance politique de la commission Laurendeau-Dunton aux politiques de protection des francophones en situation minoritaire
}

\author{
Christophe Traisnel \\ Université de Moncton
}

Anne-Andrée Denault Chaire de recherche sur la francophonie Université d'Ottawa

\section{Résumé}

Nous souhaitons montrer dans ce texte que l'État canadien semble, en fait, s'être prévalu d'une sorte de « droit d'inventaire » quant aux débats, aux réflexions et aux propositions formulés dans le cadre de la Commission royale d'enquête sur le bilinguisme et le biculturalisme, en choisissant de s'attarder davantage à trouver des solutions pour régler la " crise " politique que traversait alors le Canada, plutôt qu'en en suivant les recommandations. Ce faisant, si les conflits linguistiques au Canada se sont peu à peu pacifiés, un problème reste en suspens : celui de la reconnaissance des francophonies canadiennes. Il ne s'agit pas de proposer ici une analyse historique de la portée et des limites des travaux de la Commission, mais plutôt de comprendre l'influence de ses travaux sur les fondements du " modèle canadien de reconnaissance » à partir desquels sont pensées 
les principales politiques destinées aux communautés francophones en situation minoritaire (CFSM). Pour ce faire, nous chercherons à cerner la place relativement modeste qu'occupe la question de la reconnaissance politique dans une littérature pourtant dense consacrée aux communautés francophones en situation minoritaire, puis nous resituerons la Commission dans les débats identitaires qui traversaient à l'époque le Canada français. Nous montrerons ensuite que le législateur canadien s'est plutôt engagé dans une politique de protection des francophones en situation minoritaire, plutôt que dans une politique de reconnaissance de ces communautés. Cela nous permettra, enfin, en menant une réflexion plus prospective sur le "modèle " canadien, de nous attarder sur les conséquences de ces politiques sur les francophonies canadiennes elles-mêmes.

\section{Abstract}

In this article, we wish to demonstrate that the Canadian government, in the context of the debates, studies and propositions formulated during the Royal Commission on Bilingualism and Biculturalism, chose to prioritize the search for solutions to the political "crisis" faced by the country at the time, instead of following the recommendations of the Commission. As a result, although the linguistic conflicts present at the time calmed down, a problem remained: the acknowledgement of the different Canadian francophonies. Our aim isn't to propose an historical analysis of the reach and limits of the efforts of the Commission, but rather to understand the influence of those efforts on the foundations of the "modèle canadien de reconnaissance" through which were conceived the main politics aimed at the "francophone communities in a minority situation". To achieve this aim, we try to identify the relatively limited place occupied by this question of the "reconnaissance politique" in the dense literature on francophone communities outside of Quebec, and then to resituate the $B \& B$ Commission in the debates regarding identity within FrenchCanada at the time. We'll demonstrate then that the Canadian legislator chose to protect francophone communities in a minority situation instead of acknowledging those communities. We'll finally open on a prospective 
discussion on the Canadian "model" and on the consequences of those politics on francophone communities outside of Quebec.

Et voilà que les Canadiens-Français, à leur tour, entrent dans le cycle du changement, des prises de conscience et des grands réaménagements. JaCQUes-Yvan MORIN ${ }^{1}$

La fin des années 1960 est marquée en Amérique du Nord par une effervescence politique et une montée du courant d'affirmation culturelle et identitaire plus largement inscrit dans le mouvement des droits civiques. Tandis qu'aux États-Unis certains groupes revendiquent la reconnaissance de leur différence et l'accession à un statut égalitaire, au Canada cette effervescence s'illustre, entre autres, par une prise de conscience politique des francophones qui mènera, par exemple, à la tenue des États généraux du Canada français, à la montée du mouvement étudiant en Acadie, aux débats entourant la Commission royale d'enquête sur le bilinguisme et le biculturalisme et aux revendications souverainistes au Québec. La question nationale se situe ainsi au cœur du politique alors que le Canada français est en pleine ébullition identitaire. De ce point de vue, le contraste est saisissant comparativement à la fin des années 2000, alors que l'espace politique canadien, à tout le moins sur la question nationale, parât largement pacifié : un souverainisme en berne au Québec, des organismes acadiens travaillant sereinement au renouvellement de la Loi sur les langues officielles au Nouveau-Brunswick et des communautés francophones en situation minoritaire qui se sont toutes inscrites dans un travail de collaboration de longue haleine avec le gouvernement fédéral en vue d'assurer leur « vitalité » par l'entremise

${ }^{1}$ Jacques-Yvan Morin, "Le fondement des États généraux : discours prononcé à l'ouverture de l'Assemblée, le 23 novembre 1967 ", L'Action nationale, vol. 57, $\mathrm{n}^{\circ} 6$ (février 1968), intitulé Les États généraux du Canada français: assises nationales 1967, p. 12, [En ligne], [http://collections.banq.qc.ca/ark:/52327/2163581]. 
des programmes publics mis en œuvre dans le cadre de la législation sur les langues officielles.

Pourtant, au regard des résultats statistiques et des rapports annuels du Commissariat aux langues officielles, cette vitalité est bien loin d'être assurée. En effet, en dépit des quarante années de politiques linguistiques issues en partie des réflexions générées à la fin des années 1960, notamment par la commission Laurendeau-Dunton qui, pourtant, ouvrait la voie à une véritable reconnaissance politique des collectivités francophones, il semble que l'un des principaux acquis de ces politiques linguistiques qui ont suivi les débats de la Commission soit plus une forme de "pacification tranquille " de la question linguistique ${ }^{2}$, qu'une tentative de solutionner le problème de l'assimilation progressive de la minorité francophone au Canada.

Nous souhaitons montrer dans ce texte que l'État canadien semble, en fait, s'être prévalu d'une sorte de " droit d'inventaire" quant aux débats, aux réflexions et aux propositions formulés dans le cadre de la Commission, en choisissant de s'attarder davantage à trouver des solutions pour régler la " crise " qu'en en suivant les recommandations. Nous entendons ici par droit d'inventaire la démarche d'un acteur politique visant à opérer un bilan global d'une action collective passée, pour accepter d'endosser certains aspects jugés positifs pour l'avenir et, au contraire, rejeter ou écarter d'autres aspects considérés comme plus problématiques ou obsolètes. Un tel regard critique caractérise habituellement le commanditaire de tout travail d'enquête confié à une commission, et les travaux de la commission Laurendeau-Dunton ne font, bien sûr, pas exception à la règle. En l'espèce, l'État canadien, dans son inventaire, semble avoir fait le choix de la pacification linguistique d'abord, plutôt que

${ }^{2}$ Christophe Traisnel, « Protéger et pacifier : la politique officielle de bilinguisme canadien face aux risques de transferts linguistiques et de contestation communautaire ", Revue internationale d'études canadiennes = International Journal of Canadien Studies, n ${ }^{\circ} 45-46$ (2012), p. 69-89, [En ligne], [http://id.erudit.org/ iderudit/1009895ar]. 
celui de la protection de la minorité de langue française, par sa reconnaissance politique en tant que communauté.

Pour ce faire, nous avons procédé à une lecture des divers rapports de la Commission. Nous nous sommes particulièrement attardés aux livres suivants : Livre I : Les langues officielles, Livre II : L'éducation, parce qu'ils traitent des communautés francophones en milieu minoritaire. Nous avons aussi étudié le rapport préliminaire de la Commission, les travaux supplémentaires commandés par celle-ci, particulièrement celui sur le fédéralisme, de même que les recommandations du Conseil consultatif des districts bilingues publié plus tardivement, en 1971. Tandis que plusieurs travaux sur la Commission portent sur le Québec comme angle d'analyse, notre corpus aborde une question souvent occultée, celle des communautés francophones en milieu minoritaire, donnant ainsi à notre travail une perspective nouvelle.

Ce faisant, l'objectif de cet article demeure modeste. Il ne s'agit pas de proposer ici une analyse historique de la portée et des limites des travaux de la Commission : nombre d'auteurs, notamment dans le numéro de cette revue, se sont saisis de ce travail. Nous souhaitons plutôt comprendre l'influence des travaux de la Commission sur les fondements du « modèle canadien de reconnaissance " à partir desquels sont pensées les principales politiques destinées aux communautés francophones en situation minoritaire (CFSM). Nous chercherons, notamment, à mieux saisir le contraste qui peut exister entre, d'une part, les conclusions de la Commission et, d'autre part, les principaux cadres de référence du modèle canadien de reconnaissance des minorités linguistiques, tel qu'il a pu s'imposer depuis ces quarante dernières années : le modèle d'un État qu'il serait possible de qualifier de " compassionnel ${ }^{3}$ ", dont l'objectif est davantage d'aider et de rassurer les francophones en situation minoritaire, que de résoudre,

3 Denis Laforgue, "Des "esprits d'État" face à la ségrégation scolaire ", Actes de la recherche en sciences sociales, vol. 4, $\mathrm{n}^{\circ} 180$ (2009), p. 64-73. 
par la reconnaissance politique des communautés, la question de leur assimilation ${ }^{4}$.

Pour ce faire, nous procéderons en quatre temps. Premièrement, nous tenterons très rapidement de cerner la place relativement modeste qu'occupe la question de la reconnaissance politique dans une littérature pourtant dense consacrée aux communautés francophones en situation minoritaire. Dans un second temps, nous reviendrons sur l'esprit même de la Commission en la resituant dans les débats identitaires qui traversaient à l'époque le Canada français, pour mieux appréhender la voie de la reconnaissance politique que ces débats paraissaient suggérer. Il s'agit, en particulier, de montrer à quel point la commission Laurendeau-Dunton paraît s'inscrire dans une volonté assez singulière de renégocier l'entente confédérative, en reconnaissant le statut égalitaire de deux principaux partenaires en tant que " peuples fondateurs ". Dans un troisième temps, nous montrerons que le législateur canadien, suite aux recommandations de la Commission et bien que faisant sien ce souci de pacification linguistique qui a conduit à la mise sur pied de celle-ci, choisit pourtant de ne pas s'engager dans le paradigme de la reconnaissance politique et semble s'inscrire dans celui, moins polémique, de "l'État compassionnel », en destinant ses politiques linguistiques non pas à une communauté " francophone " ou " canadienne-française " reconnue en tant que telle, mais plutôt à des groupes linguistiques spécifiques (parents francophones, clientèles de centres communautaires, ayants droit, anglophones en situation minoritaire, etc.) en fonction d'une logique aux visées plus intégratrices que particularistes. Dit autrement, l'État canadien choisira plutôt d'aménager, au sein même de la société canadienne, une place aux francophones au moyen de politiques linguistiques offertes par l'État-providence, plutôt que de réformer l'État-nation canadien en vue d'une reconnaissance politique de la francophonie canadienne participant, en tant qu'acteur autonome, aux institutions de l'État. Enfin, dans un quatrième temps, en nous

${ }_{4}$ Traisnel, « Protéger et pacifier ». 
livrant à une réflexion plus prospective $\mathrm{du}$ " modèle » canadien, nous nous attarderons aux conséquences de ces politiques sur les francophonies canadiennes elles-mêmes ainsi que sur la question identitaire et l'imagination d'un "faire communauté » en français au Canada.

\section{De la reconnaissance politique d'une minorité à la vitalité d'une pratique linguistique en situation minoritaire : abor- der l'enjeu de la place du français au Canada}

\section{L'enjeu linguistique : du débat sur l'identité à la mesure de la vitalité}

Un rapide survol des recherches consacrées à la minorité francophone du Canada permet de dresser le portrait des transformations qui ont touché les approches consacrées à cet objet. Issue de questionnements sur les transformations du Canada français, notamment depuis les travaux de la Commission royale d'enquête sur le bilinguisme et le biculturalisme, cette littérature s'est d'abord consacrée aux dimensions historique, littéraire et linguistique du "fait français " au Canada ${ }^{5}$, pour opérer, dans les années 1970, une mutation vers des préoccupations plus sociopolitiques portant sur des thématiques qui deviendront, par la suite, centrales dans l'analyse de la "vitalité " des communautés francophones au Canada : l'éducation d'abord, puis la culture et les médias, la justice, la santé, le développement économique, l'immigration ${ }^{6}$. Notons que, dans le même temps, le

\footnotetext{
5 Fernand Harvey, «Le champ de recherche sur les communautés francophones minoritaires au Canada : sa structuration, ses orientations ", Francophonies d'Amérique, n 14 (automne 2002), p. 11-27, [En ligne], [http://id.erudit.org/ iderudit/1005180ar].

${ }^{6}$ Edmund A. Aunger, «Les communautés francophones de l'Ouest : la survivance d'une minorité dispersée ", dans Joseph Yvon Thériault (dir.), Francophonies minoritaires au Canada : l'état des lieux, Moncton, Éditions d'Acadie, 1999, p. 283304; André Magord, Rodrigue Landry et Réal Allard, «La vitalité ethnolinguistique de la communauté franco-terreneuvienne de la péninsule de Port-au-Port : une étude comparative ", dans André Magord (dir.), Les Franco-Terreneuviens de la péninsule de Port-au-Port : évolution d'une identité franco-canadienne, Moncton, Université de Moncton, Chaire d'études acadiennes, 2002, p. 197-227; Jean-Pierre
} 
gouvernement fédéral va peu à peu s'investir dans ces domaines et, par la mise en ouvre de politiques sectorielles, imposer son tempo et ses cadres de référence à la littérature consacrée à la francophonie canadienne ${ }^{7}$.

Désormais, ce « champ d'études " sur la francophonie canadienne en situation minoritaire (il existe un réseau, des revues, des instituts, des chaires et des programmes de recherche qui lui sont consacrés) se trouve structuré autour de trois grandes thématiques très inégalement couvertes par les recherches. Il existe toujours, en effet, une perspective identitaire qui poursuit les questionnements, dans des débats parfois très vifs concernant les fondements sociaux et historiques d'une "appartenance " et d'une représentation de type communautaire des enjeux linguistiques ${ }^{8}$. En général, les travaux historiques traitant de la francophonie canadienne se situent dans cette perspective. Quel

Wallot (dir.), La gouvernance linguistique : le Canada en perspective, Ottawa, Les Presses de l'Université d'Ottawa, 2005.

7 Daniel Bourgeois, Wilfrid Denis, Donald Dennie et Marc L. Johnson, La contribution des gouvernements provinciaux et territoriaux à l'épanouissement des communautés francophones en milieu minoritaire : un premier bilan et quelques prospectives, Moncton, Institut canadien de recherche sur les minorités linguistiques, 2006, [En ligne], [http://www.icrml.ca/fr/recherches-et-publications/publications-de-1$\mathrm{icrml} /$ item/8409-la-contribution-des-gouvernements-provinciaux-et-territoriauxa-l-epanouissement-des-communautes-francophones-en-milieu-minoritaireun-premier-bilan-et-quelques-prospectives]; Association des universités de la francophonie canadienne et Association internationale des études québécoises, La recherche sur la francophonie dans les Amériques : rapport final soumis au Secrétariat aux affaires intergouvernementales canadiennes du gouvernement du Québec, mars 2006, [En ligne], [http://www.aieq.qc.ca/documents/0000/0189/rapport. recherche.francophonie.pdf]; Marc L. Johnson et Paule Doucet, Une vue plus claire : évaluer la vitalité des communautés de langue officielle en milieu minoritaire, Ottawa, Commissariat aux langues officielles, 2006.

8 Marcel Martel, Le deuil d'un pays imaginé : rêves, luttes et déroute du Canada français : les rapports entre le Québec et la francophonie canadienne, 1867-1975, Ottawa, Les Presses de l'Université d'Ottawa, 1997; Monica Heller, Linguistic Minorities and Modernity: A Sociolinguistic Ethnography, London, Longman, 1998; Joseph Yvon Thériault, Faire société : société civile et espaces francophones, Sudbury, Éditions Prise de parole, 2007; Michel Bock, Quand la nation débordait les frontières: les minorités françaises dans la pensée de Lionel Groulx, Montréal, Éditions Hurtubise $\mathrm{HMH}, 2004$. 
rôle joue ou peut jouer la dimension linguistique dans le projet de «faire société » au Canada ${ }^{9}$ ? Une deuxième perspective s'inscrit dans la foulée de l'intervention de l'État et du développement de politiques de reconnaissance du français au Canada: Loi sur les langues officielles, mise en place de politiques publiques, développement de mécanismes visant à susciter le développement d'une véritable gouvernance communautaire ou linguistique, plans d'action, feuilles de route ont ainsi considérablement nourri une perspective juridico-politique tentant, tout à la fois, d'évaluer non seulement la portée, mais aussi les limites des interventions gouvernementales au regard des défis et des enjeux rencontrés par les communautés. Ce sont le statut de la langue comme celui des communautés qui sont questionnés : quelle est la juste place des francophones au Canada? Dans quelle mesure la « lutte pour la reconnaissance » entamée par la minorité francophone a-t-elle porté fruit ${ }^{10}$ ? Les modalités offertes par le pouvoir politique (droits, politiques publiques, mécanismes de collaboration, gouvernance) sont-elles adaptées aux demandes de reconnaissance ${ }^{11}$ ? Ces questionnements s'inscrivent, évidemment, dans les réflexions menées au Canada sur la notion de reconnaissance ou de ses dimensions juridiques et politiques ${ }^{12}$, et plus particulièrement sur les

9 Thériault, Faire société.

10 François Charbonneau, «L'avenir des minorités francophones du Canada après la reconnaissance ", Revue internationale d'études canadiennes = International Journal of Canadian Studies, n 45-46 (2012), p. 163-186, [En ligne], [http://id.erudit. org/iderudit/1009900ar].

11 José Woehrling, "Conflits et complémentarités entre les politiques linguistiques en vigueur au Québec, au niveau fédéral et dans le reste du Canada ", dans Pierre Noreau et José Woehrling (dir.), Appartenances, institutions et citoyenneté, Montréal, Wilson et Lafleur, 2005, p. 295-319; Will Kymlicka et Alan Patten (dir.), Language Rights and Political Theory, New York, Oxford University Press, 2003; Linda Cardinal et Luc Juillet, "Les minorités francophones hors Québec et la gouvernance des langues officielles au Canada ", dans Wallot (dir.), La gouvernance linguistique, p. 157-176; Marcel Martel et Martin Pâquet (dir.), Légiférer en matière linguistique, Québec, Les Presses de l'Université Laval, 2008.

12 Charles Taylor, Multiculturalisme : différence et démocratie, Paris, Aubier, [1992] 1994 ; James Tully, Strange Multiplicity: Constitutionalism in the Age of Diversity, Cambridge, Cambridge University Press, 1995; Will Kymlicka, Multicultural 
"luttes pour la reconnaissance " entreprises par les francophones au Canada, et pour lesquelles la question de leur achèvement se pose avec acuité ${ }^{13}$.

Une troisième perspective s'intéresse moins aux questions portant sur la reconnaissance et les représentations, qu'à celle de l'évaluation des tendances sociodémographiques de la pratique du français au Canada. Cette perspective "vitalitaire » domine largement le prisme des études sur la francophonie au Canada et cherche à repérer les principaux facteurs qui influencent la pratique d'une langue en situation minoritaire, telle que le français au Canada ${ }^{14}$, avec un objectif : essayer d'enrayer le processus de transfert linguistique ou d'assimilation qui touche la plupart des communautés francophones en situation minoritaire. De fait, la question centrale n'est plus celle de la place politique, mais celle de la dimension sociodémographique d'un groupe linguistique donné, dans un contexte marqué par le bilinguisme officiel.

Ce bref survol permet de saisir le glissement qui s'est opéré dans cette littérature, passant des préoccupations politiques centrées sur la reconnaissance et la représentation vers des questionnements d'ordre démographique, axés sur la mesure de pratiques linguistiques visant, in fine, à mesurer la vitalité du groupe linguistique concerné. Dans cette perspective, ce sont essentiellement les cadres de référence étatiques qui se sont imposés. Plus de quarante années de législation

Citizenship: A Liberal Theory of Minority Rights, Oxford. Oxford University Press, 1995; Michel Bastarache, "La place des droits linguistiques dans l'ordre juridique au Canada ", dans Martel et Pâquet (dir.), Légiférer en matière linguistique, p. 339-358.

13 Charbonneau, "L'avenir des minorités francophones du Canada après la reconnaissance ".

${ }^{14}$ Rodrigue Landry et Réal Allard, «Diglossia, Ethnolinguistic Vitality, and Language Behavior ", International Journal of the Sociology of Language, $n^{\circ} 108$ (1994), p. 1542; Charles Castonguay, "Vitalité du français et concentration des francophones : un bilan 1971-2001 ", Francophonies d'Amérique, n 20 (automne 2005), p. 15-24, [En ligne], [http://id.erudit.org/iderudit/1005333ar]; Anne Gilbert (dir.), Territoires francophones: études géographiques sur la vitalité des communautés francophones $d u$ Canada, Québec, Éditions du Septentrion, 2010. 
sur les langues officielles et de reconnaissance du bilinguisme ont imprimé leur marque sur ce champ. En s'investissant dans la question de la vitalité de la minorité francophone et en cherchant à l'évaluer, le pouvoir public a généré le besoin de poursuivre les recherches. Ces dernières ont non seulement intégré dans leur analyse les acronymes officiels de CLOSM (communautés de langue officielle en situation minoritaire) et de CFSM (communautés francophones en situation minoritaire), mais elles se sont partiellement spécialisées dans une sphère, celle qui étudie les possibilités de financement offertes par le législateur dans les domaines considérés comme particulièrement cruciaux s'agissant de l'avenir des communautés : santé, culture, éducation, immigration et développement économique.

Cet investissement de l'État fédéral et le processus de « capacitation " (empowerment) qu'a généré cet investissement au sein de la minorité francophone $\mathrm{a}$, bien sûr, conduit à une réinterprétation de la notion même de pouvoir, en fonction de ce contexte plus propice et encadré. Il ne s'agit plus de savoir si un projet de reconnaissance politique reste possible en s'interrogeant sur " le pouvoir en Acadie " ou ailleurs, par exemple, dans une perspective autonomiste qui sonne désormais comme une douce utopie, mais si les organismes communautaires disposent de marges de manœuvre suffisantes pour assurer et mettre en œuvre les services publics qui leur ont été confiés au moyen d'une forme de gouvernance qui les associe, parfois étroitement, aux prises de décision publique. Il apparaît que le Canada a progressivement renoncé aux débats portant sur la reconnaissance politique de sa minorité linguistique, à savoir l'institutionnalisation d'un groupe par l'aménagement de son autonomie politique au sein d'une société plus large, au profit de la mise sur pied de politiques de reconnaissance ne visant plus à accorder une place à un acteur collectif donné et reconnu en tant que tel, mais à multiplier les interventions publiques en vue de définir un ensemble de possibilités politiques à destination 
de divers acteurs, à charge pour eux, avec l'aide du pouvoir public, de contribuer à l'épanouissement ou à la protection de groupes donnés ${ }^{15}$.

Ce faisant, les termes mêmes des débats contemporains portant sur la place des francophones en situation minoritaire au Canada semblent bien éloignés de ceux qui, pourtant, avaient cours dans les années 1960 .

\section{Le contexte des années 1960 : la langue au cour du politique}

Dans les années 1960, la dimension politique et les questionnements à propos de la représentation et de la reconnaissance de la nation canadienne-française sont bien plus qu'aujourd'hui au cœur des débats, et le continuum des solutions politiques pour contrer la peur de l'assimilation parait beaucoup plus étendu. C'est dans ce contexte d'effervescence politique que le gouvernement de Lester B. Pearson met en place la commission Laurendeau-Dunton.

Les transformations qui s'opèrent durant les années 1950 et au début des années 1960 apportent, en effet, avec elles une nouvelle conception des rapports entre francophones et anglophones, qui se fonde sur le désir de négocier avec la majorité, de la contraindre à la reconnaissance de l'égalité entre les deux peuples, tout en revendiquant une identité distincte. Cette nouvelle représentation découle, entre autres, du constat que la nation canadienne-française se trouve dans une situation de sous-développement économique et politique malgré l'action assidue portée par un mouvement clérico-nationaliste depuis plusieurs décennies et désormais en pleine mutation ${ }^{16}$. De plus, la

15 Christophe Traisnel et Darius Bossé, «La "communauté linguistique française" du Nouveau-Brunswick dans l'article 16.1 de la Charte canadienne des droits et libertés : entre politiques de reconnaissance et reconnaissance politique d'une communauté linguistique au Canada ", Francophonies d'Amérique, n 37 (printemps 2014), p. 39-63.

${ }^{16}$ Depuis le milieu des années 1950, le mouvement canadien-français perd des membres. Les associations francophones traditionnelles peinent à recruter et à amasser les fonds nécessaires au soutien et à la promotion du français. Les caisses des associations se vident tandis que le discours de la survivance et de la foi protectrice de la langue résonne de moins en moins aux oreilles des Canadiens 
situation objective des francophones se dégrade. Le recensement de 1961 constitue, tout à la fois, un coup dur et un coup de fouet pour le mouvement canadien-français traditionnel (voir le tableau 1). Les données du recensement, reprises dans le rapport préliminaire de la Commission, montrent, selon les commissaires, une "diminution alarmante » de la proportion des francophones au pays en raison non seulement des transferts linguistiques, mais aussi de la baisse du taux de natalité. De plus, les enquêtes sur la représentation des francophones dans l'administration fédérale lancent le bal des appels à un État fédéral plus juste et représentatif des Canadiens français. Ces données viennent nourrir les revendications du mouvement néonationaliste, mais ne découragent pas ceux qui pensent plutôt que l'heure est venue de renégocier une entente politique entre nations (les intellectuels Jacques-Yvan Morin, André Laurendeau et Claude Ryan, notamment). C'est dans ce contexte que s'amorcent parallèlement deux entreprises : l'une émanant de la société civile, les États généraux du Canada français, et l'autre, portée par l'État fédéral sous l'égide du premier ministre Lester B. Pearson, la Commission royale d'enquête sur le bilinguisme et le biculturalisme. La contemporanéité de ces deux processus et la similitude des problématiques abordées les rendent mutuellement perméables, dans un contexte marqué par le désir politique de reconnaissance des Canadiens français. Les États généraux sont ainsi le reflet d'une époque où la majorité francophone du Québec découvre dans sa gouverne un instrument susceptible de lui permettre de rétablir l'équilibre et de régler les différends linguistiques qu'elle et les autres collectivités francophones estiment subir depuis trop longtemps. Cette prise de conscience ne sera évidemment

français et particulièrement ceux du Québec, qui amorcent leur Révolution tranquille. Le nationalisme axé sur la spiritualité ou le messianisme est en perte de vitesse tandis que se mettent en place des revendications d'ordre politique et constitutionnel qui prennent assises sur un désir de renouveler la Confédération de 1867 (Anne-Andrée Denault, Divergences et solidarité : une étude sociopolitique des rapports entre le Québec et les francophones d'Amérique, thèse de doctorat (science politique), Université d'Ottawa, 2013). 
pas sans effet sur l'évolution des débats au sein de la Commission qui, elle aussi, tente de dégager des solutions à ce problème, qui se transforme peu à peu en crise politique majeure.

\section{Tableau 1}

Répartition, en pourcentage, selon la langue maternelle, de la population du Canada et de chacune des provinces en 1961

\begin{tabular}{|l|c|c|c|c|}
\hline & Anglais & Français & Autre & Total \\
\hline Canada & 58,5 & 28,1 & 13,4 & 100 \\
\hline Terre-Neuve & 98,6 & 0,7 & 0,7 & 100 \\
\hline Île-du-Prince-Édouard & 91,4 & 7,6 & 1,0 & 100 \\
\hline Nouvelle-Écosse & 92,3 & 5,4 & 2,3 & 100 \\
\hline Nouveau-Brunswick & 63,3 & 35,2 & 1,5 & 100 \\
\hline Québec & 13,3 & 81,2 & 5,5 & 100 \\
\hline Ontario & 77,5 & 6,8 & 15,7 & 100 \\
\hline Manitoba & 63,4 & 6,6 & 30,0 & 100 \\
\hline Saskatchewan & 69,0 & 3,9 & 27,1 & 100 \\
\hline Alberta & 72,2 & 3,2 & 24,6 & 100 \\
\hline Colombie-Britannique & 80,9 & 1,6 & 17,5 & 100 \\
\hline Yukon & 74,3 & 3,0 & 22,7 & 100 \\
\hline Territoire du Nord-Ouest & 35,6 & 4,3 & 60,1 & 100 \\
\hline
\end{tabular}

Source : Recensement de 1961 (extrait de l'étude de Ronald L. Watts, Les sociétés pluriculturelles et le fédéralisme, Étude de la Commission royale d'enquête sur le bilinguisme et le biculturalisme, vol. 8, Ottawa, Imprimeur de la reine, 1971, p. 97).

\section{Les travaux de la Commission : jalons pour une reconnais- sance politique du Canada français}

La décennie 1960 se trouve largement marquée par la dimension politique de l'enjeu linguistique. C'est la question nationale et, plus spécifiquement, la place du Canada français dans la fédération canadienne qui se trouve au cour des réflexions. À la lumière des 
débats qui précèdent la Commission et des réflexions et solutions proposées, le gouvernement Pearson semble résolument s'orienter sur la voie d'une reconnaissance culturelle, identitaire et, par extension, politique des constituants du Canada français.

\section{L'instauration de la Commission}

Longuement attendue par le Canada français, la Commission est instituée dans un contexte de fédéralisme coopératif, pour reprendre la typologie de Richard Simeon et Ian Roberson, qui encourage la négociation et la recherche d'ententes entre les groupes linguistiques ${ }^{17}$. En effet, les années d'après-guerre, loin d'avoir mené à un rapprochement entre ces deux groupes (comme certains l'auraient souhaité) semblent plutôt révéler une polarisation grandissante entre le Québec, majoritairement francophone, et le reste du Canada, menaçant l'unité canadienne. Selon les commissaires, la Commission a été mise sur pied pour « [...] examiner les griefs formulés de plus en plus vigoureusement par les Canadiens français et, en particulier, le Québec. C'est le Canada français qui, par ses porte-parole, se déclare insatisfait de l'état des choses actuel et assure qu'il est victime d'inégalités inacceptables ${ }^{18}{ }$. Sensible à cette réalité politique, le

17 Pour ces auteurs, le fédéralisme au Canada est souvent pensé sous l'angle des conflits entre les régions et les groupes linguistiques et comme un système où le pouvoir est divisé entre plus de deux ordres de gouvernement, qui ont chacun leurs domaines de juridiction. Leur approche contextuelle permet de comprendre que l'époque propre à la Commission était caractérisée par une ouverture à la négociation, qui sera remplacée par un fédéralisme compétitif au début des années 1970 (Richard Simeon et Ian Robinson, "The Dynamics of Canadian Federalism ", dans James Bickerton et Alain-G. Gagnon (dir.), Canadian Politics, $4^{\mathrm{e}}$ éd., Peterborough, Broadway Press, 2004, p. 101-126).

${ }_{18}$ Commission royale d'enquête sur le bilinguisme et le biculturalisme (ci-après, Commission $\mathrm{BB}$ ), Rapport préliminaire de la Commission royale d'enquête sur le bilinguisme et le biculturalisme, Ottawa, Imprimeur de la reine, 1965, p. 15. Laurendeau écrira dans son journal personnel : "Les Canadiens français du Québec traversent présentement une phase d'autocritique : ils sont insatisfaits de leur politique provinciale, de leur enseignement, du rôle qu'ils jouent dans l'économie de leur province, etc., et leur jugement sur la Confédération fait 
premier ministre de l'époque, Lester B. Pearson, accepte, contrairement à l'ancien gouvernement de John Diefenbaker, de mettre en place une commission royale qui sera chargée de faire une vaste consultation pancanadienne. S'échelonnant de 1963 à 1969, elle sera présidée par deux intellectuels reconnus, issus des "deux solitudes »: André Laurendeau, éditeur du quotidien francophone de Montréal Le Devoir, et Davidson Dunton, président de l'Université Carleton.

Lors de sa première allocution en 1963 en tant que coprésident, André Laurendeau soulignera la portée politique de cette initiative gouvernementale : "Le gouvernement canadien, en instituant la Commission royale d'enquête sur le bilinguisme et le biculturalisme, a clairement indiqué qu'il avait la certitude d'une urgence - d'une urgence qui peut mettre en cause l'existence même du Canada ${ }^{19}$. " La Commission aura donc pour objectif de pacifier les conflits, d'éviter une crise qui pourrait déchirer le Canada, en « rétablissant » la notion d'égalité entre les deux " peuples fondateurs ». Dès l'instauration de la Commission et la détermination de son mandat, il semble que la reconnaissance politique soit la voie privilégiée pour régler les différends existant entre les peuples majoritaire et minoritaire, notamment par l'affirmation de la dualité canadienne. Comme le soulignent les commissaires dans le premier chapitre du rapport préliminaire, le mandat de la Commission sera "de faire enquête et rapport sur l'état présent du bilinguisme et du biculturalisme, et de recommander les mesures à prendre pour que la Confédération canadienne se développe d'après le principe de l'égalité entre les deux peuples qui l'ont fondée, compte tenu de l'apport des autres groupes ethniques à l'enrichissement culturel du Canada, ainsi que les mesures à prendre pour sauvegarder cet apport ${ }^{20}$ ». Lors des premières audiences, les commissaires se rendent rapidement compte que cette égalité entre les peuples

partie de tout cela. Le mouvement actuel est donc, par conséquent, moins antibritannique que plusieurs nationalismes du passé " (André Laurendeau, Journal tenu pendant la Commission royale d'enquête sur le bilinguisme et le biculturalisme, Montréal, VLB éditeur, 1990 p. 73).

19 Commission BB, Rapport préliminaire, p. 170.

${ }^{20}$ Ibid., p. 172. Nous soulignons. 
ne fait pas l'unanimité chez le groupe majoritaire. Ils choisissent pourtant de la défendre dans le rapport préliminaire : « [...] certains ont déclaré qu'elle n'est inscrite ni dans la constitution ni dans l'histoire du Canada. [...] Il est vrai que le principe de l'égalité culturelle est une orientation de base : c'est une interprétation légitime, parmi d'autres, de la constitution et de l'histoire ${ }^{21}$. " Les commissaires font donc le choix d'adhérer à une certaine version de la théorie du pacte et de chercher à revoir les fondements du pays ${ }^{22}$.

Dès les premières consultations auprès de la population, les commissaires remarquent, d'ailleurs, que l'urgence des débuts s'est muée en une véritable crise politique et sociale. Selon les commissaires, « [...] tout se passe comme si l'état de choses établi en 1867 et jamais gravement remis en question depuis, était pour la première fois refusé par les Canadiens français du Québec ${ }^{23}$ ". À cet effet, les commissaires soulignent « que le problème se trouve actuellement posé de la façon la plus radicale : les Canadiens français vont-ils se concevoir euxmêmes comme un Canada français centré sur le Québec, mais vitalement solidaire de sa diaspora, ou comme une société exclusivement québécoise? C'est un problème qu'ils résoudront d'abord eux-mêmes, mais très certainement en fonction des attitudes adoptées par le Canada de langue anglaise ${ }^{24}$ ». Ainsi, les membres de la Commission soulignent l'importance du rôle de la majorité dans l'avenir du pays et, plus implicitement, de son ouverture face à une refonte des rapports entre les peuples.

Dans ce même esprit se préparent les États généraux du Canada français, composés de représentants francophones venant des différentes régions du Canada. Ceux-ci se réuniront en 1967 pour discuter des enjeux concernant l'avenir du français au pays et d'une stratégie

${ }^{21}$ Ibid., p. 15.

22 Se référer à l'étude de Ramsay Cook effectuée dans le cadre de la Commission royale d'enquête sur le bilinguisme et le biculturalisme, L'autonomie provinciale, les droits des minorités et la théorie du pacte, 1867-1921, vol. 4, Ottawa, Imprimeur de la reine, 1969.

${ }^{23}$ Commission BB, Rapport préliminaire, p. 5.

${ }^{24}$ Ibid., p. 111. 
pour renégocier l'entente confédérative. Comme le déclare JacquesYvan Morin lors de la séance d'ouverture, le 23 novembre 1967 :

Nous avons toujours défendu avec opiniâtreté nos droits collectifs, en particulier nos libertés linguistiques et scolaires, mais presque partout dans ce pays on nous a réduits à les quémander. Et voici que nous découvrons qu'il existe une liberté plus large que personne ne peut nous ravir : le droit de disposer de nous-mêmes en tant que peuple $[\ldots]^{25}$.

Encore une fois, la question se pose sous l'angle des rapports de nation à nation, d'une relecture de l'entente entre peuples impliquant une reconnaissance politique des groupes concernés, et non simplement de l'aménagement de droits linguistiques. Suivant cette approche, les collectivités francophones à l'extérieur du Québec, bien que parties prenantes de ce Canada français, de ce "nous " évoqué par Jacques-Yvan Morin, sont reconnues comme des entités à part entière, avec leur unicité et leurs besoins spécifiques ${ }^{26}$.

\section{La reconnaissance politique du peuple canadien-français : au cour des réflexions de la Commission}

En écho aux débats qui agitent les États généraux au même moment, la question de la reconnaissance politique du Canada français, par l'entremise d'un Canada constitué de deux peuples fondateurs, se trouve au cœur des réflexions des commissaires. Cette conception repose, selon eux, sur la base juridique de la Confédération de 1867, qui fait du Québec le "foyer de la nation canadienne-française ". Selon Pearson, le mandat de la Commission s'appuie sur le double postulat de l'existence d'un Canada uni par un régime commun et de l'égalité des deux peuples ${ }^{27}$. "Nous croyons résumer le mandat en disant que son idée-force, c'est le bilinguisme et le biculturalisme (l'anglais et le français) et en ajoutant immédiatement que cette idée-

\footnotetext{
Morin, "Le fondement des États généraux », p. 14.

Denault, Divergences et solidarité.

27 Commission BB, Rapport préliminaire, p. 15.
} 
force s'exerce sur un milieu où existe un multiculturalisme de fait multiculturalisme que l'on ne nous invite pas à supprimer le plus vite possible (ce qui serait du melting pot), mais au contraire à respecter et à sauvegarder, sans toutefois lui donner une reconnaissance officielle ${ }^{28}$."

Ce postulat de l'égalité ne renvoie pas à l'égalité individuelle, mais plutôt, selon les commissaires : " [...] il s'agit pour nous du citoyen en tant qu'il participe à l'une des deux cultures; c'est l'égalité de l'anglophone et du francophone en tant que tels quelle que soit leur origine ethnique ${ }^{29}$. "Sur la notion de biculturalisme, Dunton déclarera lors des audiences préliminaires de la Commission, les 7 et 8 novembre 1963 :

Au Canada le mot «biculturalisme " évoque la notion de deux grandes cultures, rattachées à l'une des deux langues principales, qui, certes, ont beaucoup en commun tout en ayant leurs qualités distinctives. Ce sera une partie importante de notre tâche d'étudier comment ces deux cultures peuvent grandir vigoureusement dans l'égalité ${ }^{30}$.

C'est ici l'illustration de la notion d' "equal partnership " (principe de l'égalité entre les deux peuples) évoquée par Laurendeau à de nombreuses reprises et qu'il défend, d'ailleurs, dans ses écrits dès les années $1940^{31}$.

${ }^{28}$ Ibid., p. 178.

29 Ibid., p. 16. Nous soulignons.

30 Ibid., p. 172. Lors d'une audience préliminaire, le président conjoint Davidson Dunton déclarait : "D'autre part, il est clair que le concept d'égalité ne peut être simple. Il y a plus de Canadiens anglophones que de francophones de telle sorte qu'une rigoureuse égalité ne peut exister au point de vue numérique ", notant ainsi au passage qu'il avait conscience de la complexité de l'application de la notion d' " equal partnership " dans une situation où le rapport de force est déséquilibré (Ibid.).

31 Cette conception se réclame de la pensée d'Henri Bourassa qui, dans les années 1910, dans un souci de préserver et de faire avancer les droits des Canadiens français proposait un cadre différent pour interpréter le pacte confédératif et formulait la théorie du pacte entre deux peuples, les Canadiens anglais et les Canadiens français, plutôt que celle du pacte entre provinces. Elle se fonde sur l'existence ou 
Dans ces débats, on reconnaît clairement que la situation des Canadiens français vivant à l'extérieur du Québec nécessite des mesures particulières tout comme celle, d'ailleurs, de la communauté anglophone du Québec. Le rapport préliminaire discute longuement des " minorités françaises ", qui revêtent, selon les commissaires, une importance à la fois réelle, car il s'agit de 850000 personnes de langue maternelle française, et symbolique pour l'avenir du Canada. Ainsi notent-ils : «[...] les minorités françaises sont déjà l'un des facteurs importants du bilinguisme à travers le pays; et le deviendront bien davantage si on leur fournit les moyens. D’un autre côté, ces minorités ont toujours été un lien entre le Québec et les autres provinces canadiennes ${ }^{32}$."

\section{Les recommandations: l'affirmation de l'existence de communautés}

Les recommandations du livre premier (Les langues officielles) appuient l'idée que l'on se trouve devant une forme (certes « asymétrique ») de reconnaissance politique de la nation canadiennefrançaise qui distingue le Québec des minorités francophones et les collectivités minoritaires entre elles, qu'elles soient anglophones ou francophones. Il s'agit, en quelque sorte, d'adapter les politiques de reconnaissance linguistique à la situation de la nation canadiennefrançaise. De ce point de vue, les commissaires recommandent, entre autres :

1 - « [...] aux provinces du Nouveau-Brunswick et de l'Ontario de déclarer d'elles-mêmes qu'elles reconnaissent l'anglais et le français comme langues officielles, et qu'elles acceptent le régime linguistique découlant de cette déclaration ${ }^{33}$ »;

la reconnaissance d'une dualité culturelle qui ne serait pas liée à un territoire en particulier et dont le Québec serait le château fort et les collectivités francophones minoritaires des avant-postes. Voir un des articles d'André Laurendeau écrit lorsqu'il était directeur de L'Action nationale, "Alerte aux Canadiens français! ", L'Action nationale, vol. 16, no 3 (novembre 1940), p. 177-203, [En ligne], [http:// collections.banq.qc.ca/ark:/52327/2163581].

32 Commission BB, Rapport préliminaire, p. 111.

33 Ibid., p. 99. 
2- «[...] que toute province canadienne dont la minorité linguistique officielle atteindra ou dépassera dix pour cent déclare qu'elle reconnaît l'anglais et le français comme langues officielles et qu'elle accepte le régime linguistique découlant de cette déclaration ${ }^{34}$ »;

3- «[...] que les provinces autres que le Québec, le NouveauBrunswick et l'Ontario reconnaissent l'usage du français et de l'anglais dans leurs assemblées législatives, et que ces provinces procurent à leurs minorités francophones les services appropriés en français ${ }^{35} »$;

4- " [...] que des districts bilingues soient créés au Canada et que par voie de négociation, le gouvernement fédéral et le gouvernement provincial concerné fixent les limites exactes de chacun $^{36} »$.

Dans les provinces du Nouveau-Brunswick et de l'Ontario, qui seraient officiellement bilingues suite aux recommandations des commissaires, ceux-ci notent : «L'instruction obligatoire impose déjà aux gouvernements provinciaux de mettre des écoles à la disposition de tous les enfants; ils seront en outre tenus d'établir des écoles de langue française partout où les jeunes francophones seront assez nombreux. [...] Dans chacune des provinces officiellement bilingues, nous aurons des réseaux parallèles et complets d'enseignement en anglais et en français, depuis les classes élémentaires jusqu'aux études supérieures ${ }^{37}$. » Et ils poursuivent à propos des districts bilingues, en notant : "C'est dans le district bilingue que l'on devrait trouver assez d'élèves pour former des classes françaises. Une école ne saurait toutefois exister isolément; on doit la concevoir dans le cadre d'un système. [...] Les écoles de la minorité doivent répondre aux mêmes exigences $^{38}$."

\footnotetext{
${ }^{34}$ Ibid., p. 102.

35 Ibid., p. 108.

36 Ibid., p. 114.

37 Ibid., p. 130.

38 Ibid., p. 130-131.
} 
Ainsi, dans la logique des districts bilingues il y a création "d'écoles françaises » vues par les commissaires comme une " innovation ${ }^{39}$ ». Les commissaires vont au-delà d'une simple reconnaissance du droit des parents de faire instruire leurs enfants en français ou en anglais. Il est ici question d'institutions d'enseignement liées à la minorité francophone puisque, comme ils le soulignent à de nombreuses reprises : «Le gouvernement du Québec s'acquitte déjà de cette obligation envers sa minorité anglophone ${ }^{40}$. "

Suite à leurs recommandations, les commissaires soulignent l'importance d'inscrire ces droits dans l'Acte de l'Amérique du Nord britannique (AANB) pour "donner l'assurance qu'ils seront respectés ${ }^{41}$ ». Entre autres, ils souhaitent l'extension de l'article 93, qui deviendrait 93a et se lirait comme suit :

Chaque province établira et soutiendra des écoles primaires et secondaires utilisant l'anglais comme unique langue d'enseignement et des écoles primaires et secondaires utilisant le français comme unique langue d'enseignement, dans les districts bilingues et les autres régions appropriées que déterminera la législation provinciale; mais aucun terme du présent article ne sera interprété comme interdisant les écoles où l'anglais et le français auraient une importance égale en tant que langues d'enseignement, ou celles qui pourraient dispenser l'enseignement dans une autre langue ${ }^{42}$.

En amendant l'article 93, les commissaires souhaitent s'assurer que les minorités francophones jouissent des mêmes droits que la minorité anglophone du Québec. Ainsi, ils poursuivent en notant que ces droits doivent être affirmés à la fois dans la Constitution canadienne et dans les statuts provinciaux ${ }^{43}$.

À plusieurs égards, les recommandations de la commission Laurendeau-Dunton impliquent une reconnaissance à la fois

39 Ibid., p. 132.

40 Ibid., p. 130.

${ }^{41}$ Ibid., p. 139.

42 Ibid., p. 140. En gras dans le texte.

${ }^{43}$ Ibid., p. 139. 
sociologique et politique des communautés. Trois éléments appuient notre propos.

Premièrement, l'affirmation du statut égal des deux langues officielles ne signifie pas uniquement une égalité dans la loi, mais aussi une égalité dans l'application de cette loi, qui prend en compte le contexte de ces collectivités. C'est dire que des mesures doivent être appliquées de façon différente selon les situations que requièrent le Québec, les anglophones du Québec et les minorités «françaises ", pour reprendre le terme des commissaires. Dans les fameuses pages bleues du rapport écrites par Laurendeau, ce dernier note, de surcroît, que la langue est " une condition nécessaire, mais insuffisante ${ }^{44}$ » au maintien intégral d'une culture. Ce qui renvoie à une notion plus "substantive » de la langue qui ne permet pas une dissociation entre langue et culture (ou communauté). La reconnaissance de deux grandes cultures (biculturalisme), qu'on associe évidemment aux deux groupes linguistiques, mais aussi à deux styles de vie et deux façons de vivre distinctes, vient renforcer cette conception. Les commissaires, et plus spécifiquement Laurendeau, affirment : "Ce fait de l'altérité, si évident quand il s'agit de la langue, si fort et si frappant quand il se manifeste concrètement au plan culturel, mais néanmoins si difficile alors à isoler et à exprimer, sera souvent illustré dans d'autres parties du rapport et dans plusieurs travaux de recherche. Les deux cultures dominantes [...] s'incarnent au Canada dans des sociétés distinctes ${ }^{45}$. " Ces deux cultures s'incarnent dans des sociétés que les commissaires définissent, dans le rapport préliminaire, comme " les formes d'organisation et les institutions qu'une population assez nombreuse, animée par la même culture, s'est données et a reçues, dont elle dispose librement sur un territoire assez vaste et où elle vit de façon homogène, selon des normes et des règles de conduite qui lui sont communes ${ }^{46}$ ". Les commissaires soulignent, par le fait même,

${ }^{4}$ Ibid., p. XxvII. Nous soulignons.

45 Ibid., p. XxiIr. Nous soulignons.

${ }^{46}$ Commission BB, Rapport préliminaire, p. 103. 
des éléments de sociétés francophones autonomes (par exemple, le Nouveau-Brunswick).

Deuxièmement, le Québec, pour une première fois, est reconnu non seulement comme une société distincte, mais également comme le foyer de la nation canadienne-française. Pearson reconnaissait en ce sens le rôle singulier du Québec et affirmait, dans un discours prononcé en 1964, «[...] l'importance particulière (de posséder ses droits propres) pour le Québec, qui est non seulement une province, mais aussi la patrie de la plupart des Canadiens de langue française, le gardien de leur langue, de leurs traditions et de leur mode de vie ${ }^{47}$ ". Cette reconnaissance sera totalement mise de côté par le gouvernement de Trudeau, qui cherchera, tout à la fois, à faire du gouvernement fédéral le " protecteur » des minorités francophones et à éteindre toute forme de nationalisme.

Troisièmement, la création de districts bilingues permet de reconnaître l'existence de communautés bien réelles, distinctes et autonomes, diversement réparties sur l'ensemble du territoire canadien, mais participant chacune à la même nation, consacrant ainsi une approche résolument collective de l'identité. Dit autrement, la reconnaissance ne peut faire l'économie des représentations identitaires qui accompagnent la pratique (et le partage) d'une langue. Pour les commissaires, et au risque de se répéter, la langue et la culture sont indissociables; elles sont des "phénomènes collectifs ». En ce sens, ils écriront : «[...] une culture exprime une communauté d'expérience et d'attitudes, et ne s'épanouit que si l'individu vit avec d'autres qui participent à cette communauté. Cela signifie qu'une culture ne sera pleinement vécue qu'au sein de la société où elle s'incarne ${ }^{48}$."

D'une certaine manière, la Commission propose au législateur non pas une suite de réformes, mais bien une refonte en profondeur du système étatique et de la société canadienne dans son ensemble en vue de conférer une juste représentation aux deux groupes

Laurendeau, Journal tenu pendant la Commission, p. 77.

48 Commission BB, Rapport, Livre 1 : Les langues officielles, p. xxxiv. Nous soulignons. 
initialement convoqués à ce travail de réflexion sur la société et l'État canadien. Au-delà du statut officiel de deux langues, ce sont bel et bien des collectivités distinctes, des nations qui sont ici désignées et visées par les recommandations. Or l'État canadien, dans le traitement de la question nationale qu'il proposera par la suite, choisira une voie bien différente.

\section{De la reconnaissance politique aux politiques de protection et de promotion linguistiques : le virage du bilinguisme et du multiculturalisme}

Pour comprendre un tel choix, il convient de mieux saisir le fond des tensions auxquelles l'État était alors confronté. Vers la fin des années 1960, l'État canadien se trouvait face à un défi singulier : d'une part, la peur de certains citoyens en situation minoritaire subissant une forme d'assimilation; d'autre part, chez le groupe majoritaire cette fois, la "peur de la peur ", ou une inquiétude au sujet des conséquences potentiellement délétères du développement des mobilisations autour de l'enjeu linguistique et du conflit politique lui-même au Canada.

La mise sur pied de la Commission visait, semble-t-il, à répondre à cette double peur et à assurer, avant même d'émettre l'idée d'une quelconque justice linguistique, une forme de pacification d'un conflit linguistique de plus en plus menaçant pour la société canadienne. Dit autrement, en instaurant la Commission, le gouvernement canadien cherchait surtout à maintenir une sorte d' " ordre public linguistique ", à même de garantir la paix sociale (c'est là, d'ailleurs, une des principales fonctions des États), par la régulation des conflits et des politiques publiques adaptées aux enjeux du moment. Cette paix linguistique impliquait de faire preuve de compréhension face aux défis rencontrés par le citoyen minorisé, en lui donnant les outils (reconnaissance, participation, collaboration) pour contrer cette minorisation et les dangers de l'assimilation. 
Devant cet enjeu, les travaux de la commission LaurendeauDunton semblaient, à bien des égards, s'inscrire dans une forme répandue de réponse aux conflits ethnolinguistiques : la prise en compte, par un aménagement institutionnel adéquat, des revendications portées par la minorité et, partant, la reconnaissance politique de cette minorité revendicatrice en tant que telle. D'ailleurs, l'étude comparative de Ronald L. Watts, entreprise dans le cadre de la Commission et visant à examiner diverses fédérations pluriculturelles dont l'expérience pouvait servir à éclairer la situation canadienne, allait dans ce sens $s^{49}$. Cette forme d'autonomie consiste en une prise en compte institutionnelle et légale, plus ou moins territorialisée, de la distinction évoquée. Elle se traduit par la mise sur pied d'une représentation politique institutionnalisée et l'aménagement des cadres de l'État national permettant à ce dernier d'intégrer la communauté ainsi distinguée. C'est, dans une large mesure, le processus qui a été suivi en Belgique où l'État national belge s'est progressivement détaché d'une approche très centralisatrice et unitariste pour adopter, grâce à un lent processus de dissociation, une forme fédérale laissant une large place à l'expression des particularismes régionaux et communautaires belges. Cette forme classique de pacification linguistique par la reconnaissance politique est, en général, le modèle qui a cours face aux revendications autonomistes rencontrées en Europe, y compris dans les contextes (en tout cas, à l'origine) très unitaires de l'Italie, du Royaume-Uni, de l'Espagne et même de la France (en NouvelleCalédonie, territoire français, on reconnaît ainsi une citoyenneté calédonienne, distincte de la citoyenneté française). Une telle reconnaissance politique se traduit donc, dans l'espace politique de l'État, par la présence d'une collectivité à part entière. Dans cette situation, c'est donc la collectivité ainsi reconnue, en fonction des pouvoirs qui lui sont conférés et des compétences qui lui sont

49 Ronald L. Watts, Les sociétés pluriculturelles et le fédéralisme, Étude de la Commission royale d'enquête sur le bilinguisme et le biculturalisme, vol. 8, Ottawa, Imprimeur de la reine, 1971. 
accordées, qui jouera un rôle central, notamment dans la définition de l'identité collective, par l'intermédiaire d'une véritable gouverne à même de prendre en charge une bonne partie de ce travail de construction identitaire, et la réalisation d'un projet de société distincte. En ce sens, la voie de l'autonomie et de la reconnaissance politique ne se traduit pas seulement par un aménagement des cadres de l'espace politique, mais bien par la présence d'un acteur nouveau, autre : le groupe minoritaire, à même d'entrer dans une forme de négociation partenariale avec la majorité, au sein d'un espace politique commun.

Même si la commission Laurendeau-Dunton reste très prudente, ses propositions s'inscrivent dans la voie d'une reconnaissance politique, territorialisée et pancanadienne de «la » communauté francophone au Canada. Tout en tentant de concilier les revendications de la minorité avec les objections et les résistances de la majorité, elle s'inscrit dans la voie d'une telle reconnaissance, notamment en déclarant que le Canada français forme une nation et que l'État québécois est l'"État national » du Canada français, en proposant l'établissement sur une base territoriale de districts bilingues dans les provinces ou les régions où un groupe linguistique atteint $10 \%$ de la population, ou en suggérant l'instauration d'institutions d'enseignement associées à chacune des langues officielles. Même modestes en ce qui a trait notamment aux solutions institutionnelles, ces propositions s'inscrivent dans l'approche autonomiste que nous avons évoquée plus haut : le " bilinguisme ", très étroitement lié au " biculturalisme ", rend à tout le moins possible l'idée d'un contrat entre partenaires et ouvre (éventuellement) la voie à une forme d'autonomie politique même si sa mise en œuvre demeure un casse-tête dans un contexte politique tel que celui du Canada des années 1960.

Malgré la prudence des commissaires, ce n'est pas cette voie-là qui a été retenue par le législateur. À ce titre, l'arrivée au pouvoir de Pierre Elliott Trudeau en 1968 constitue une étape nouvelle dans les réflexions sur l'identité canadienne et la reconnaissance de la nation canadienne-française, dont le rapport Laurendeau-Dunton constituait 
en quelque sorte une forme atténuée, ou modeste, de cristallisation. Le choix est alors celui, non de l'aménagement, mais plutôt de la confrontation avec les thèses indépendantistes, tout en tentant de faire de l'État fédéral à la fois le défenseur de l'unité nationale et celui de la cause du français partout au Canada. Le 17 octobre 1968, dans une déclaration à propos de la Loi sur les langues officielles, Pierre Elliott Trudeau présente son approche de la diversité canadienne et de la place, au sein de cette diversité, de l'enjeu linguistique. Opposant assez clairement dans ce discours l'homogénéité et l'uniformité de certains pays occidentaux au monde contemporain ouvert à la diversité, Trudeau évoque le même dilemme qui se pose au Canada et la manière dont le gouvernement a choisi de le régler :

L'exemple le plus important de cette diversité est sans aucun doute l'existence des deux principaux groupes linguistiques, qui sont tous deux assez importants et riches en ressources matérielles et intellectuelles pour résister aux forces de l'assimilation. Dans le passé, nos institutions publiques n'ont pas reflété adéquatement cette réalité, qui est à la base même de notre pays [...].

Dans toutes les parties du pays, au sein des deux groupes linguistiques, il y a ceux qui réclament l'uniformité. Ce sera plus simple et moins cher, disent-ils. Dans le cas de la minorité française, l'isolement est prescrit comme étant nécessaire à la survivance. Nous ne devons jamais sous-estimer la force ou la durabilité de ces appels aux sentiments humains profonds.

Il est certain que ces arguments se fondent sur la peur, sur une conception étroite de la nature humaine, et sur une évaluation défaitiste de notre habileté à modifier notre société et ses institutions pour répondre aux demandes de ses citoyens.

[...] Nous avons rejeté cette façon de voir notre pays. Nous croyons en deux langues officielles et en une société pluraliste, non seulement comme une nécessité politique mais comme un enrichissement. Nous voulons vivre dans un pays où les Canadiensfrançais $[s i c]$ peuvent choisir de vivre parmi les Canadiens-anglais [sic] et où les Canadiens anglais peuvent choisir de vivre parmi les Canadiens-français [sic] sans renoncer à leur héritage culturel. 
Ceux d'entre nous qui ont une certaine expérience des difficultés et des possibilités de ce choix en connaissent le risque. Mais nous sommes convaincus que nous devons, en tant que pays et en tant qu'individus, le couriir ${ }^{50}$.

Cette déclaration, faut-il le rappeler, est formulée dans un contexte où les travaux de la Commission ne sont pas encore achevés et où celle-ci a perdu l'un de ses éminents commissaires, qui portait avec lui une vision du Québec et de la nation canadienne-française à l'opposé de celle de Trudeau. Les travaux de la Commission en seront grandement teintés, comme le montre la lecture des Recommandations du Conseil consultatif des districts bilingues, un texte publié en mars 1971. Tandis que du point de vue des commissaires, au début des travaux, la notion de reconnaissance est au cœur de la solution, il n'en est pas de même pour Trudeau, qui refuse de reconnaître le Québec comme société distincte privilégiant une société contemporaine qui favorise le choix des individus en matière linguistique. Trudeau formulera un autre refus, lié au précédent : pas plus que le Québec n'est distinct, il ne constitue un quelconque foyer national du Canada français dans le cadre d'un dualisme culturel et linguistique reconnu. Un tel foyer existe, pour Trudeau, c'est le Canada lui-même, dans le cadre d'une approche certes bilingue, mais également multiculturelle ${ }^{51}$. Bien que les commissaires et Trudeau semblent en accord sur la nécessité de procéder à une réforme constitutionnelle et sur l'instauration de politiques de bilinguisme qui, selon Trudeau, permettront d'unir le pays et de freiner ce qu'il nomme «l'exaltation du fait national ${ }^{52}$ ", les réformes proposées par

50 Pierre Elliott Trudeau, Déclaration à propos de la Loi sur les langues officielles, le 17 octobre 1968, [En ligne], [https://www.collectionscanada.gc.ca/primeministers/ h4-4066-f.html] (17 juillet 2014).

51 En ce sens, Trudeau refusera de reconnaître la légitimité des État généraux du Canada français puisqu'ils œuvrent en dehors des cadres politiques établis et ne sont donc pas représentatifs.

52 Pour Trudeau, une nation fondée sur des « attributs ethniques ", en parlant du projet souverainiste au Québec, aboutit inévitablement à l'intolérance (Pierre Elliott Trudeau, Le fédéralisme et la société canadienne-française, Lasalle, Hurtubise, 1967). 
les commissaires different considérablement de celles qui seront mises en place par le gouvernement. Sous l'influence d'une nouvelle génération de décideurs politiques canadiens issus du Québec (Pierre Elliott Trudeau, bien sûr, mais également Jean Marchand ou Gérard Pelletier, notamment), l'idée qui prévaut est, en effet, celle du caractère passablement dépassé de la notion de biculturalisme prôné par la Commission. Dans un monde de plus en plus ouvert et dans une société canadienne de plus en plus marquée par la diversité culturelle et les revendications concernant la reconnaissance qu'implique cette diversité, l'idée de l'État-nation est « absurde " et les visées autonomistes "rétrogrades ${ }^{53}$ ". Ce second refus des propositions concernant la reconnaissance politique avancées par les commissaires se traduit par l'abandon de la notion de deux « peuples fondateurs".

Pour cette nouvelle élite, la solution passe certes par la reconnaissance d'un bilinguisme pancanadien, essentiellement fondé sur la liberté de choix linguistique des individus, quitte à mettre en place des politiques sociales susceptibles de garantir effectivement cette liberté linguistique, notamment dans les contextes particuliers où des groupes de citoyens se trouveraient en "situation minoritaire ", mais également par la reconnaissance du multiculturalisme comme fondement de l'identité de la société canadienne contemporaine.

Comme l'affirme Trudeau en 1962, «[...] il faut divorcer les concepts d'État et de nation, et faire du Canada une société vraiment pluraliste et polyethnique ${ }^{54}$ ». La question linguistique ne se pose plus sous l'angle des rapports entre communautés, comme en témoignent les rencontres régionales organisées dans le cadre des travaux de la Commission, mais concerne désormais les politiques publiques ${ }^{55}$.

53 Voir Pierre Elliott Trudeau, "La nouvelle trahison des clercs ", Cité libre, $13^{\mathrm{e}}$ année, $\mathrm{n}^{\circ} 46$ (avril 1962), p. 3-16, [En ligne], [http://collections.banq.qc.ca/ ark:/52327/2225396].

54 Ibid., p. 15.

55 Les consultations publiques tournaient autour de trois questions principales: "Ces deux peuples, l'anglophone et le francophone, peuvent-ils et veulent-ils vivre 
On s'éloigne ainsi définitivement d'une sortie de crise privilégiant le modèle autonomiste. Comme le note Réjean Pelletier, la politique du multiculturalisme " [...] nie complètement le biculturalisme et ne cherche nullement à reconnaître la caractéristique spécifique du groupe culturel québécois : ce dernier devient un groupe culturel parmi tant d'autres ${ }^{56}$ ». En somme, comme les autres groupes, les francophones du Québec peuvent (et doivent) compter sur la loi canadienne pour garantir la vitalité linguistique de leur société.

À ce titre, le changement n'a pas que pour conséquence de reconnaître, à côté de la culture française, l'apport d'autres cultures comme le voulaient les commissaires, mais conduit plutôt à une dissociation fondamentale entre la langue, soumise à des politiques de reconnaissance linguistique à travers le bilinguisme, et la culture, soumise à d'autres politiques de reconnaissance, culturelle cette fois. À cette dissociation entre langue et culture que voulaient éviter les commissaires, s'ajoute l'idée qu'il n'y a pas d'accommodement possible entre la volonté de construire une nation québécoise, de plus en plus présente à la fin des années 1960, notamment en raison des revendications linguistiques, et celle de construire une nation canadienne, à partir des années d'après-guerre. Comme le remarque Réjean Pelletier, «durant la période [...] 1957-1984, le gouvernement fédéral s'emploie à sauvegarder son rôle dans l'ensemble du pays et à préserver l'unité canadienne. Ce thème de l'unité va s'imposer, au début de cette période avec Diefenbaker et à la fin avec Trudeau, dans une vision "uniformisatrice" où s'estompe le rêve de la dualité canadienne ${ }^{57}$ ». L'égalité des peuples se mue en une simple dualité linguistique accompagnée du multiculturalisme.

ensemble? À quelles conditions nouvelles? Et ces conditions, sont-ils prêts à les accepter?" (Commission BB, Rapport préliminaire, p. 148. En italique dans le texte).

56 Réjean Pelletier, "Constitution et fédéralisme ", dans Manon Tremblay, Réjean Pelletier et Marcel R. Pelletier (dir.), Le parlementarisme canadien, $2^{\mathrm{e}}$ éd. revue et augmentée, Québec, Les Presses de l'Université Laval, 2000, p. 45.

57 Ibid., p. 69. 
Comme l'ont fort bien montré plusieurs auteurs, dont Kenneth McRoberts ${ }^{58}$, il ne s'agissait plus d'aménager, au sein de l'espace politique canadien, une place à une collectivité culturellement et linguistiquement distincte, mais de construire un "nouveau Canada " susceptible de répondre aux craintes de disparaître et d'être assimilé exprimées pendant des décennies par les minorités de langue française du Canada, sans passer par la proposition, délétère, de l'autonomie. En d'autres termes, les solutions à la crise ne se posent désormais plus dans la perspective de la reconnaissance d'une collectivité désignée et définie (le "Canada français »), mais plutôt à travers une multiplicité de politiques portant sur la langue. Une langue désincarnée, simple outil de communication, mais aussi élément de distinction pour un Canada à la recherche d'une identité nationale. En effet, comme le souligne Éric Waddell, la Commission royale sur le développement national des arts, des lettres et des sciences, créée en 1949, avait conclu que le Canada était menacé par l'américanisation ${ }^{59}$. La politique du bilinguisme vient donc ici répondre à cette peur identitaire du Canada anglais en se distinguant des États-Unis par l'affirmation d'un pays bilingue. Intéressant paradoxe de penser que cette politique a peut-être mieux servi la majorité anglophone que la nation canadienne-française, qui cherchait reconnaissance et réparation.

\section{Pacifier plutôt que protéger : quarante ans de législation linguistique fondée sur le bilinguisme officiel}

En dissociant biculturalisme et bilinguisme, le législateur rend ainsi impossible toute forme de reconnaissance politique d'une collectivité minoritaire francophone particulière au Canada, d'abord parce que la langue devient un "patrimoine commun ", national, ensuite, parce

58 Kenneth McRoberts, Misconceiving Canada: The Struggle for National Unity, Toronto, Oxford University Press, 1997.

59 Éric Waddell, «L'État, la langue et la société : les vicissitudes du français au Québec et au Canada ", dans Alan Cairns et Cynthia Williams (dir.), Les dimensions politiques du sexe, de l'ethnie et de la langue au Canada, Ottawa, Approvisionnements et services Canada, 1986, p. 95. 
que le multiculturalisme introduit l'idée que les distinctions culturelles sont multiples. Cette approche "pancanadienne » des langues, singulièrement éloignée de l'esprit de la Commission sur le bilinguisme et le biculturalisme, sera largement consacrée par les politiques linguistiques canadiennes mises en place à partir des années 1970. Le bilinguisme officiel devient ainsi au Canada un des outils d'affirmation nationale, qui est un des grands enjeux politiques des décennies d'après-guerre, mais également, et peut-être surtout, un instrument de canalisation (et de traitement) des revendications linguistiques multiples et le principal lieu d'expression des organismes communautaires francophones. L'État fédéral devient alors le principal acteur d'une "pacification linguistique " menée en concertation avec les organismes communautaires canadiens, invités à collaborer à leur élaboration et leur mise en œuvre. Comment se traduit cette politique de "pacification linguistique "?

D'abord, par la volonté de répondre aux craintes relatives à l'assimilation et aux inégalités entre groupes linguistiques, en adoptant, en 1969, la Loi sur les langues officielles, abrogée et remplacée par une nouvelle loi en 1988, puis modifiée en 2005 après l'adoption d'un projet de loi privé émanant du Sénat. Pierre Trudeau avait, en 1968, bien cerné la peur issue des "injustices passées », et la nécessité de répondre à cette peur par "l'habileté [du Canada] à modifier [la société canadienne] et ses institutions pour répondre aux demandes de ses citoyens $s^{60} »$.

Le renforcement du "régime linguistique ${ }^{61}$ » de 1969 à nos jours consacre le français comme l'une des deux langues officielles du Canada. Une officialisation qui se traduira par l'égalité de statut des deux langues dans toute une série de domaines liés aux institutions et aux services publics fédéraux, et le droit, reconnu à chaque Canadien, d'utiliser la langue de son choix dans ces domaines. Cette législation sera par la suite renforcée, d'une part, avec l'ajout, en

${ }^{60}$ Trudeau, Déclaration à propos de la Loi sur les langues officielles.

${ }^{61}$ Bastarache, «La place des droits linguistiques dans l'ordre juridique au Canada ». 
1988, de la partie VII, modifiée en 2005 et consacrant l'obligation légale pour le gouvernement de prendre des " mesures positives" visant à assurer un statut égal au français et à l'anglais dans la société canadienne et, d'autre part, par l'intervention des tribunaux et le développement d'une interprétation des textes plutôt favorables aux communautés de langue officielle en situation minoritaire et aux droits linguistiques dont ils bénéficient ${ }^{62}$. Une telle législation consacre le rôle de "champion " joué non seulement par le gouvernement fédéral, mais également par les tribunaux dans la promotion des langues officielles et de l'égalité linguistique. Dans le cadre de cette législation, les «situations minoritaires » se trouvent dans la ligne de mire du législateur, et les politiques linguistiques mises en œuvre consistent essentiellement à renforcer ou à garantir la "vitalité linguistique " de ces communautés de langue officielle en situation minoritaire, qu'il s'agisse des francophones en situation minoritaire ou des anglophones en situation minoritaire au Québec. Quant à la Loi constitutionnelle de 1982, elle ne donne aucun statut particulier au Québec, notamment en matière linguistique. Les dispositions linguistiques visent les francophones hors Québec et les anglophones du Québec, tout comme les dispositions nouvelles de l'article 16.1 de la Charte canadienne des droits et libertés. Adopté après l'échec de l'Accord de Charlottetown en 1992 suite à une entente bilatérale entre le gouvernement fédéral et le gouvernement du NouveauBrunswick, cet article consacre la reconnaissance des communautés " française » et " anglaise " du Nouveau-Brunswick, sans d'ailleurs les circonscrire ni même les définir ${ }^{63}$.

Autre instrument de pacification linguistique : l'adoption de plusieurs politiques linguistiques dans les domaines jugés stratégiques pour la vitalité des communautés de langue officielle en situation minoritaire. Sous l'impulsion des différents programmes d'action

${ }^{62}$ Ibid.

63 Traisnel et Bossé, «La "communauté linguistique française” du Nouveau-Brunswick dans l'article 16.1 de la Charte canadienne des droits et libertés". 
gouvernementaux (politiques ministérielles, Plan d'action pour les langues officielles, feuilles de route gouvernementales consacrées à la dualité linguistique, etc.), se développent des politiques de collaboration avec les divers organismes communautaires dans les secteurs de l'éducation, de la culture, des médias, de la santé et de l'immigration. Même si le gouvernement n'hésite pas à intervenir en ces matières de son propre chef et sans préavis, ces politiques sociales sont souvent menées en concertation avec les organismes communautaires et constituent autant de possibilités politiques qui sont proposées aux acteurs communautaires et à partir desquelles, en collaboration avec les ministères fédéraux concernés et les gouvernements provinciaux, se développent plusieurs services publics où la langue joue un rôle central. Bien qu'il ne faille pas négliger les capacités d'intervention des communautés elles-mêmes, l'investissement du gouvernement dans ces secteurs, en soutien aux initiatives communautaires, a permis la création d'écoles et de commissions scolaires francophones, de radios communautaires, d'une presse communautaire francophone, de festivals, d'événements culturels en français, de centres de soins et d'hôpitaux francophones dynamisant singulièrement les divers milieux minoritaires tant au Québec (anglophones) que dans le reste du Canada (francophones) et inscrivant peu à peu les principaux acteurs communautaires dans une démarche de partenariat et de négociation permanente avec le gouvernement fédéral. Une négociation par laquelle les conflits potentiels sont réglés, non pas d'abord dans l'espace politique, mais par la voie judiciaire et l'arbitrage des tribunaux. Même si la solution judiciaire (tout comme d'ailleurs la mobilisation politique d'une communauté) est particulièrement onéreuse, tant en ressources financières qu'en ressources humaines ou matérielles, elle tend à être privilégiée après que les démarches et les négociations avec les pouvoirs publics ont été épuisées. Il semble qu'elle se substitue à toute forme de mobilisation politique des communautés ou à tout débat de fond sur la portée et les limites du modèle canadien de reconnaissance des minorités et, notamment, du régime linguistique constitué autour du bilinguisme. 
Parallèlement se développe un nouveau milieu associatif moins politique et consacré, dans chaque secteur, à la gestion des services offerts et à la poursuite, avec les différents paliers gouvernementaux, d'une collaboration dans la mise en œuvre et la pérennisation de ces services.

Enfin, le législateur a également prévu plusieurs mécanismes permettant de vérifier si les mesures adoptées par les institutions fédérales en matière de dualité linguistique sont appliquées, l'objectif étant non seulement de favoriser partout au Canada les progrès du bilinguisme, mais également de contribuer positivement à la vitalité des communautés de langue officielle en situation minoritaire. C'est ainsi que la loi a institué un Commissariat aux langues officielles qui a pour mission de

[...] prendre, dans le cadre de sa compétence, toutes les mesures visant à assurer la reconnaissance du statut de chacune des langues officielles et à faire respecter l'esprit de la présente loi et l'intention du législateur en ce qui touche l'administration des affaires des institutions fédérales, et notamment la promotion du français et de l'anglais dans la société canadienne ${ }^{64}$.

Le Commissariat joue, en quelque sorte, le rôle de vigile linguistique au service des minorités. Son rôle est renforcé par l'intervention d'un autre mécanisme, plus contraignant pour le gouvernement : les tribunaux. Le recours aux tribunaux a d'ailleurs été largement facilité par l'instauration, en 1971, du Programme de contestation judiciaire (transformé en Programme d'appui aux droits linguistiques en 2009), permettant aux organismes communautaires (francophones hors Québec et anglophones du Québec) de financer leurs contestations judiciaires lorsqu'elles estiment que, manifestement, un gouvernement ou une institution ne respecte pas ses obligations linguistiques. Ainsi, la contestation qui s'exprime le plus souvent

${ }^{64}$ Gouvernement du Canada, Loi sur les langues officielles, [1988, ch. 38, sanctionné le 28 juillet 1988], art. 56, sur le site Web de la législation (Justice), [http://lawslois.justice.gc.ca/fra/lois/O-3.01/20021231/P1TT3xt3.html]. 
dans la francophonie canadienne n'est plus d'abord d'ordre politique, faisant appel à la mobilisation des communautés concernées, mais une contestation judiciaire, grâce à laquelle les conflits sont tranchés par des arbitrages venant des institutions étatiques fédérales et en fonction du droit existant.

\section{Le modèle canadien : le rejet de l'autonomie et la promo- tion d'une approche résolument compassionnelle de la question linguistique}

Ce processus législatif, entamé en 1969, a surtout eu pour effet de conférer des droits individuels en matière linguistique et a également permis de développer des mécanismes de contrôle sous la responsabilité des gouvernements provinciaux, dans la mise en œuvre de la législation linguistique. Ce faisant, il a conduit le Canada vers une voie bien distincte de la voie autonomiste évoquée précédemment et pourtant privilégiée par la commission Laurendeau-Dunton.

$\mathrm{Si}$, dans le contexte européen, la résolution des conflits linguistiques est généralement venue d'une transformation de l'État-nation et de l'institutionnalisation, qui ont conduit à la reconnaissance politique de communautés à part entière (par exemple, en Italie, en Allemagne, en Belgique, en Grande-Bretagne, en Espagne et, dans une moindre mesure, en France, dans un contexte où l' "Europe des régions " conduit les États les plus centralisés à aménager une place à leurs minorités par le renforcement des autonomies locales), la situation est bien différente au Canada. Celui-ci a inventé son propre modèle de résolution des conflits linguistiques, et c'est finalement l'État-providence plus que l'État-nation qui joue le premier rôle ${ }^{65}$. Cette voie canadienne, singulière, compassionnelle, consiste non pas à reconnaître une nouvelle collectivité, mais à prendre en charge la résolution de la question linguistique.

65 Traisnel, « Protéger et pacifier ». 
Face aux craintes exprimées par les communautés quant aux taux élevés de transferts linguistiques, l'État canadien répond en se présentant comme principal protecteur des citoyens canadiens en situation de fragilité. Reconnaissant d'emblée deux langues officielles, il met en place des politiques sociales permettant aux citoyens en situation minoritaire de bénéficier de mesures de protection adaptées afin de corriger de potentielles inégalités. Dans chaque domaine jugé essentiel au maintien de la vitalité des deux langues officielles, l'administration fédérale a tenté d'intervenir, en concertation avec les organismes communautaires et les usagers concernés, en vue de donner corps à la législation sur le bilinguisme. La société canadienne s'est donc peu à peu enrichie de services dont la gestion est partiellement confiée aux citoyens concernés par ces services : commissions scolaires, établissements culturels ou de santé, universités, centres d'accueil pour immigrants francophones, etc. Directement associés à la gestion et à l'entretien de ces services, les citoyens en question peuvent ainsi contribuer à la définition des programmes et à l'adaptation des services aux besoins changeants des usagers, et même contester devant les instances compétentes (Commissariat aux langues officielles, tribunaux) les éventuels manquements aux obligations linguistiques des différentes gouvernes. Une telle approche, très compassionnelle, oblige l'État à assurer, à l'égard de populations dont la fragilité est reconnue, une protection légitime. Partant, elle rend également obsolète toute forme de reconnaissance politique des communautés minoritaires. En obligeant l'État à pourvoir aux besoins des communautés et à leur protection, la législation linguistique canadienne mutualise les risques liés à l'assimilation, et elle conduit de facto les populations en situation minoritaire à s'intégrer dans la grande collectivité nationale canadienne.

Depuis les années 1960, il semble que le "modèle canadien " des politiques de reconnaissance soit arrivé à pacifier les conflits linguistiques, en multipliant les mécanismes institutionnels permettant une médiation dans les éventuels conflits entre représentants communautaires et gouvernes (intervention du juge, rôle du commis- 
saire aux langues officielles, Programme de contestation judiciaire). C'est là le succès majeur de ce modèle. Pour autant, ce modèle est-il parvenu à garantir une protection satisfaisante aux communautés francophones au point d'en garantir la pérennité? Rien n'est moins sûr, au regard des chiffres toujours alarmants des « taux de transferts linguistiques " partout au Canada, à l'exception du Québec et du Nouveau-Brunswick.

En 1940, Laurendeau écrivait : «Ainsi donc, il apparaît que nos minorités seront justement traitées, que le Canada sera reconnu comme un peuple bi-ethnique dans la mesure où un Québec bien orienté s'imposera par sa puissance ${ }^{66}$. " Près d'une vingtaine d'années plus tard, le mandat de la Commission sera " de faire enquête et rapport sur l'état présent du bilinguisme et du biculturalisme, et de recommander les mesures à prendre pour que la Confédération canadienne se développe d'après le principe de l'égalité entre les deux peuples qui l'ont fondée ${ }^{67}$ ". Les commissaires notaient dès lors : "C'est l'heure des décisions et des vrais changements; il en résultera soit la rupture, soit un nouvel agencement des conditions d'existence ${ }^{68}$. " Il semblerait qu'une troisième voie se soit dessinée, plus subtile, plus indicible, celle d'une assimilation " tranquille ", pacifiée, malgré les efforts et les bonnes volontés des principaux protagonistes de ces politiques linguistiques, tant dans les différentes fonctions publiques concernées qu'au sein des organismes communautaires, qui œuvrent quotidiennement au renforcement de la vitalité de leur communauté.

Plus de quarante ans après la remise des conclusions de la Commission, force est de constater que le rêve d'un Canada biculturel s'est largement évanoui, et que le Canada français tel qu'il a été imaginé pendant plusieurs décennies est devenu un Canada imaginaire. Bien loin d'une reconnaissance des communautés, ce sont plutôt des « situations minoritaires " qui sont

${ }^{66}$ Laurendeau, «Alerte aux Canadiens français! ", p. 179-180.

${ }^{67}$ Commission BB, Rapport préliminaire, p. 172. Nous soulignons.

${ }^{68}$ Ibid., p. 125. 
Tableau 2

Taux de transfert linguistique vers l'anglais* de la population francophone au Canada

\begin{tabular}{|l|c|c|}
\hline & $\mathbf{2 0 0 1}$ & $\mathbf{2 0 0 6}$ \\
\hline Québec & $1,0 \%$ & $1,1 \%$ \\
\hline Canada moins Québec & $35,1 \%$ & $39,3 \%$ \\
\hline Colombie-Britannique & $72,8 \%$ & $72,0 \%$ \\
\hline Alberta & $64,5 \%$ & $69,0 \%$ \\
\hline Saskatchewan & $67,5 \%$ & $74,4 \%$ \\
\hline Manitoba & $50,1 \%$ & $55,5 \%$ \\
\hline Ontario & $36,9 \%$ & $41,8 \%$ \\
\hline Nouveau-Brunswick & $9,7 \%$ & $11,2 \%$ \\
\hline Nouvelle-Écosse & $41,7 \%$ & $48,3 \%$ \\
\hline Île-du-Prince-Édouard & $46,8 \%$ & $50,7 \%$ \\
\hline Terre-Neuve-et-Labrador & $46,8 \%$ & $50,7 \%$ \\
\hline Territoires du Nord-Ouest & $55,1 \%$ & $67,9 \%$ \\
\hline Yukon & $53,8 \%$ & $54,8 \%$ \\
\hline Nunavut & --- & $47,9 \%$ \\
\hline
\end{tabular}

* Le taux de transfert linguistique vers l'anglais établit le pourcentage des personnes de langue maternelle française qui parlent le plus souvent l'anglais à la maison.

Sources : Statistique Canada, Le portrait linguistique en évolution, Recensement 2006 : 97-555-XIF au catalogue. En ligne sur le site du Secrétariat à la politique linguistique, Gouvernement du Québec, "La dynamique des langues en quelques chiffres : tableaux ", [http://www.spl.gouv.qc.ca/documentation/ rapportssondagesstatistiques/dynamiquedeslangues/tableaux/] (15 août 2013).

reconnues, à travers l'officialisation très technique de deux langues, aux espaces qui s'entrecroisent et entrent parfois encore en conflit. Il est, en effet, bien difficile dans le Canada contemporain de trouver une quelconque trace institutionnelle ou législative des francophonies canadiennes en tant que collectivités distinctes et reconnues telles. L'effort réitéré des organismes 
communautaires comme des principaux ministères concernés par le dossier des langues officielles pour définir ces communautés par la production récurrente de profils ou de portraits, tous plus différents les uns que les autres, atteste l'indécision identitaire et le caractère passablement introuvable de ces communautés, qui n'existent encore que grâce à l'effort obstiné des "faiseurs d'identité ", ou des militants de la langue qui, partout au Canada, continuent d'imaginer ces communautés et de travailler à leur reconnaissance politique. Dans une telle situation, la question qui reste en suspens est celle de savoir si la voie de la pacification linguistique sans reconnaissance de l'identité du groupe, de ce qui le distingue, bref sans reconnaissance politique ${ }^{69}$, est à même de garantir la pérennité de ces francophonies canadiennes, et si ces " taches de rousseur " sur le visage de l'Amérique, comme l'écrit si bien Zachary Richard, n'ont pas, tout simplement, vocation de disparaître un jour.

69 Sur la politique de la différence, voir Taylor, Multiculturalisme : différence et démocratie. 